\title{
Equation of state of quark-gluon plasma using a simple phenomenological model
}

\author{
Yogesh Kumar ${ }^{1, a}$ \\ ${ }^{1}$ Department of Physics, Deshbandhu College, University of Delhi, Kalkaji, New Delhi-110019, India
}

\begin{abstract}
The equation of state (EoS) of quark-gluon plasma (QGP) using a phenomenological model is studied in which finite value of quark mass is modified as effective mass. The effective mass of these quasiparticle generated due to the interaction of quarks and gluons with the surrounding matter in the medium. The model results provide EoS of QGP which are in good agreement and found almost similar results to the earlier theoretical results. This model is successfully applied to the description of the properties of quark-gluon plasma created in the collision of nucleons. Thus, the effective mass of quark shows the useful information to study the EoS of QGP in high energy heavy-ion collisions.
\end{abstract}

\section{Introduction}

The physics of heavy-ion collisions provide an excellent tool to produce nuclear matter in laboratory at very high temperature and energy density $[1,2]$. Quantum chromo-dynamics (QCD), the fundamental theory of strong interaction provides a qualitative and quantitative insight into a wealth of remarkable phenomena observed in heavy-ion collision. The theory indicates possible phase transition from a confined state to a deconfined state in the early universe [3]. A new state of matter is formed by this type of phase transition called the quark-gluon plasma (QGP) [4-7]. The search for experimental evidence of new state of matter in heavy-ion collision is still going on.

The results in the collisions of heavy nuclei at relativistic heavy-ion collision (RHIC) at BNL and large hadron collision (LHC) at CERN at very high energy density and temperature have come out as a new physics with these accelerators. The new physics search in the study of QGP resolve many unsolved query by deep involvement of theoreticians and experimentalists. Researcher believe that QGP exists for a few microseconds after the big-bang that has thrown down a great challenge to the search of QGP in these experiments. So one of the most fascinating issue is the detection of QGP.

Although unlimited efforts have been taken with the help of experimental and theoretical high energy groups, our understanding of QGP is still not up to mark $[8,9]$. Yet the theoretical understanding in the evolution of interacting system has improved considerably since the early studies in this direction. Moreover, the equation of state is required to describe the properties of QGP in central collisions of heavy massive nuclei. Since long physicists have been used a simple bag model to investigate and describe the properties of QGP [10, 11].

ae-mail: yogesh.du81@gmail.com 
Many authors have worked in the limit of zero chemical potential which is considered as a valid approximation for the study of QGP in heavy-ion collision experiments at BNL (RHIC) and CERN (LHC). Even Lattice QCD calculations also corresponds to zero or small value of chemical potential for the QGP EoS. Therefore, in this recent report, we use an effective quark mass in place of thermal quark mass using a quasiparticle model. Using this effective mass, we try to observe the behaviour of QGP EoS.

This model is therefore used as quasiparticle model which might be better improve to the Lattice QCD simulation results to produce equation of state of quark-gluon plasma. The effectice quark mass is considered as linear function of square of current mass, coupling of thermal and current mass and square of thermal mass. Our study is based on earlier model where it has been shown that how the temperature dependent quark mass effects the EoS of QGP in heavy-ion collisions.

Thus, the paper is presented as: In Section 2, we briefly discuss the theoretical model. In Section 3 , we explain the equation of state of QGP. The results are presented in Section 4. Finally, we conclude in Section 5.

\section{The theoretical model}

The detection of QGP plays a vital role in high energy field. Especially the equation of state has become one of the attractive and important signature of QGP. This indirect signature of QGP might be helpful in describing the behaviour of QGP. Therefore, it is considered as one of the important properties of QGP. There are many theoretical models which are thermodynamically consistent with perturbative QCD and non-perturbative QCD calculations [12, 13].

Peshier et al. have studied that QGP is composed of quasiparticles and mass of these particles are temperature dependent [14-16]. Earlier the calculation of free energy have been performed at zero quark mass with the effect of curvature term [17]. Further Kumar et al. [18] have modified the calculation at finite thermal quark mass. Now, we extend the previous work by using modified effective quark mass of quasiparticles and it is defined in Ref. [19]. The effective quark mass created in heavy-ion collisions is considered as linear function of square of current mass, coupling of thermal and current mass and square of thermal mass. In quasiparticle models [14-16], a massive system of noninteracting quasiparticles is described where mass of these quasiparticles depend only on temperature. The quasiparticles acquire mass due to the interaction of quarks and gluons with the surrounding matter [20-23]. The effective mass of these quasiparticles is given by Ref. [19],

$$
M_{e f f}^{2}=m_{c}{ }^{2}+\sqrt{2} m_{c} m_{q}+m_{q}^{2},
$$

where $m_{c}$ is the current mass of the quark and $m_{q}$ is the thermal mass of the quark $[18,24]$.

In order to match the Lattice QCD results, the suitable parametrization factors are incorporated. So our model is thermodynamically self consistent to describe QGP EoS. The above value of finite quark mass is given as $[18,24]$ :

$$
m_{q}^{2}(T)=\left(4 \pi \alpha_{s}\right) \gamma_{q} T^{2}
$$

where $\alpha_{s}$ is strong coupling constant and it is defined as [17, 18, 24];

$$
\alpha_{s}=\frac{4}{\left(11 N_{C}-2 N_{f}\right) \ln \left(1+\frac{k^{2}}{\Lambda^{2}}\right)},
$$

and,

$$
k=\left[\frac{\gamma N^{\frac{1}{3}} T^{2} \Lambda^{2}}{2}\right]^{\frac{1}{4}} .
$$


The number of color and quark flavors are taken three. Also all the parameters are suitably defined in the Ref. [18]. The parametrization factor $\gamma$ is used as r.m.s. value which is just like the Reynold's number. We use $\gamma_{q}=1 / 6$ [18] and $\gamma_{g}=0.02 \gamma_{q}$ so that it is suitably fit into the Lattice QCD calculations.

\section{Equation of state of QGP}

To find the equation of state of QGP, we first define free energy equation for quarks, gluons and interface term. The appropriate modification is done in the free energy term for quarks with the help of effective mass of quark using Ref. [18, 19]. It is defined as:

$$
F_{q}=-T g_{q} \int \rho_{q}(k) \ln \left[1+e^{-\left(\sqrt{M_{e f f}^{2}+k^{2}}\right) / T}\right] d k
$$

The above equation is defined for fermions (quarks). In the same manner, we can define the free energy equation for bosons (gluons) which is given as,

$$
F_{g}=T g_{g} \int \rho_{g}(k) \ln \left[1-e^{-\left(\sqrt{m_{g}^{2}+k^{2}}\right) / T}\right] d k
$$

The density of state for quarks and gluons is represented as $\rho_{q}(k)$ and $\rho_{g}(k)$. The $g_{q, g}$ is quarks and

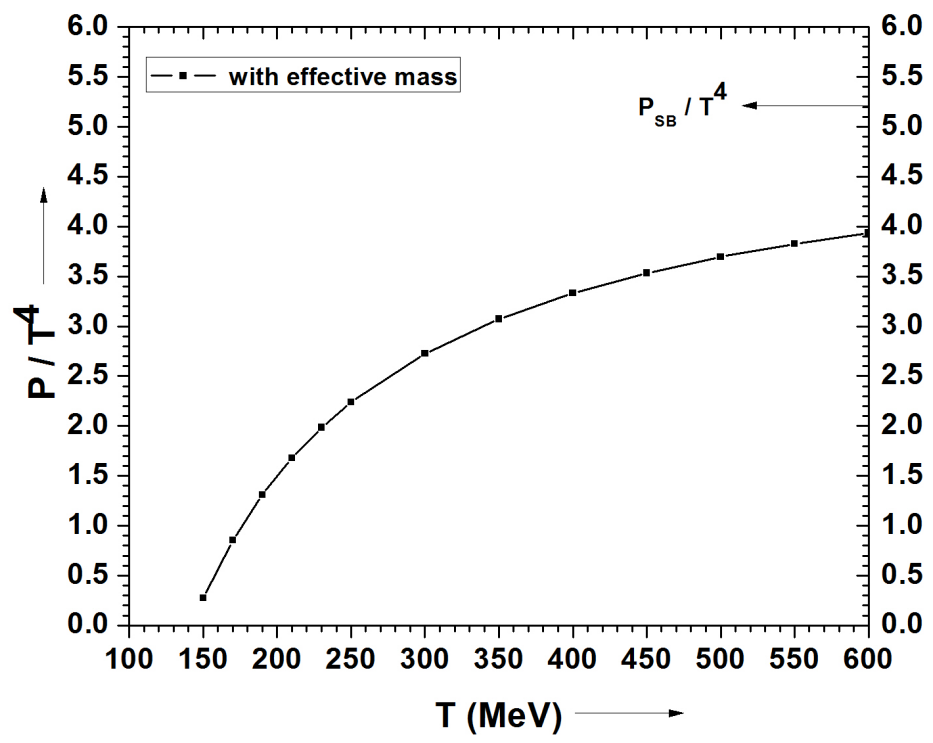

Figure 1. The pressure $\left(P / T^{4}\right)$ with respect to temperature $(T)$ is shown

gluons degenracy factor. The value of this factor is taken from Ref. [25]. In the framwork of the same model, we define the essential term as interfacial free energy. The interfacial energy is used in place of bag constant pointed out by Ref. [17]. It is taken as:

$$
F_{\text {interface }}=\frac{1}{4} R^{2} T^{3} \gamma .
$$




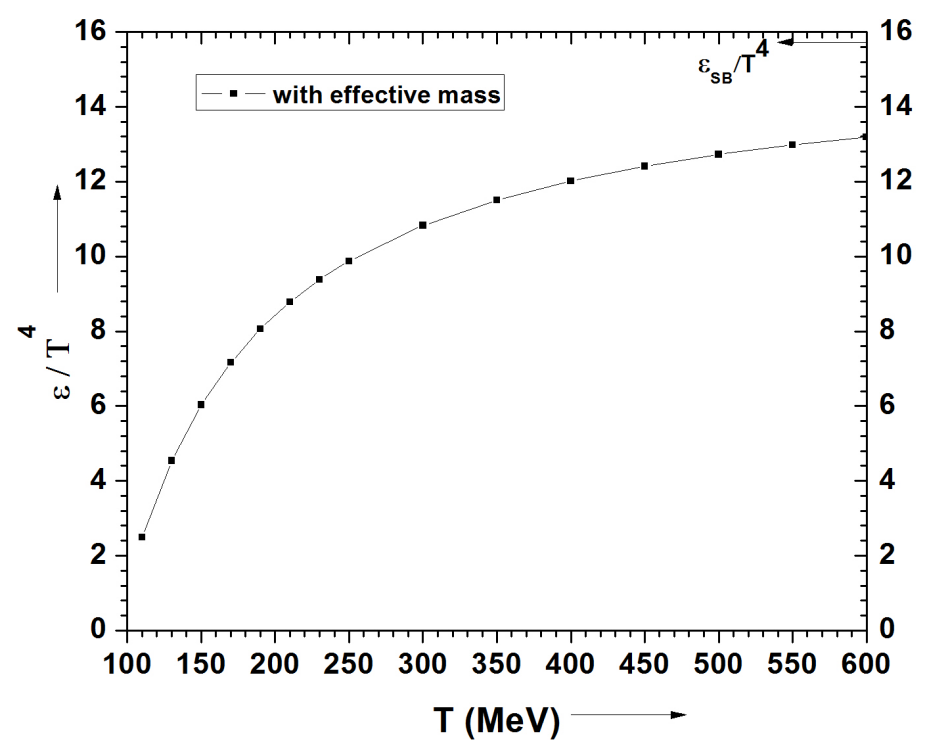

Figure 2. The energy density $\left(\varepsilon / T^{4}\right)$ with respect to temperature $(T)$ is plotted

Where the size of QGP bubble is represented by symbol $R$. Using equations 5, 6 and 7, we can easily calculate total free energy $F_{\text {total }}$ :

$$
F_{\text {total }}=F_{q=u, d, s}+F_{\text {gluon }}+F_{\text {interface }} .
$$

The above total free energy can be useful to describe the evolution of quark-gluon plasma.

Now we can compute the equation of state as $P, \varepsilon, S$ and $C_{S}^{2}$ (Square) using total free energy. It is used as Ref. [25]:

$$
P=-\left(\frac{d F_{\text {total }}}{d v}\right)
$$

The total pressure $P$ is composed of different terms like quarks, gluons and interface mentioned above. Then, energy density is also computed with the help of total pressure [25],

$$
\varepsilon=T \frac{d P}{d T}-P \text {. }
$$

Similarly we can calculate the entropy and speed of sound as,

$$
S=\frac{d P}{d T}
$$

and

$$
C_{S}^{2}=\frac{d P}{d \varepsilon}
$$

The adjustment of such parameters have taken care accordingly. The parameters are nicely fit using this model. We also compare the results obtained from the evolution of free energy. Finally 


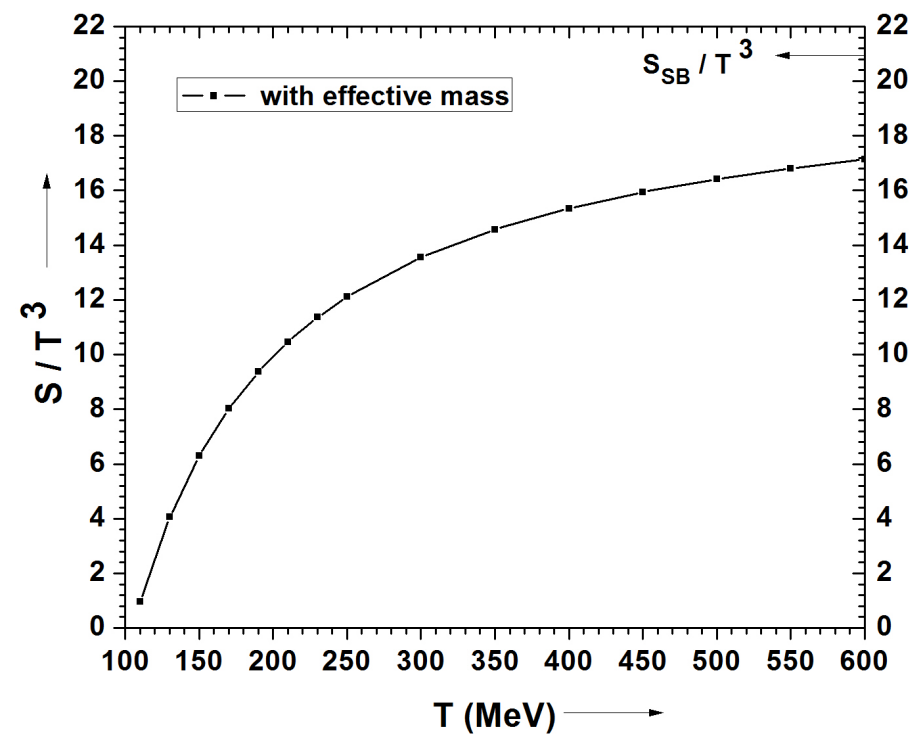

Figure 3. The entropy $\left(S / T^{3}\right)$ with respect to temperature $(T)$ is shown

these relation can be used to calculate $\operatorname{EoS}$ as $P / T^{4}, \varepsilon / T^{4}, S$ and $C_{S}^{2}$ (Square). These relations are thus applicable in exploring the equation of state of quark-gluon plasma using effective mass of quark. At the end, our findings are useful and exactly similar to our recent work. The developments in these theoretical result are indeed likely match with the recent results of Lattice QCD.

\section{Results}

In this section, we provide the results of EoS of QGP with the help of a simple phenomenological model. The different plots of EoS such as pressure $\left(P / T^{4}\right)$, energy density $\left(\varepsilon / T^{4}\right)$, entropy $(S)$ and speed of sound $\left(C_{S}^{2}\right)$ with temperature $(\mathrm{T})$ is shown in the figures.

The Figure [1] and [2] shows the pressure and energy density with respect to the temperature. Both the curves are exponentially increases and approach towards Stefan-Boltzmann (SB) limit. In both Figures, results are little decrease as comparison to our earlier results [25]. The results are not deviated much with the effective quark mass and hence almost same as our earlier results [25]. These outputs also match well to the Lattice calculation results particularly in the high temperature limit $\mathrm{T} \sim 600 \mathrm{MeV}$.

Further in Figure [3], we plot the graph between entropy and temperature. Also the plot of speed of sound with temperature is shown in figure [4]. In these Figures, it is found that the results are lying almost in the same range of our previous work. We noticed that the effective value of quark mass does not deviate much from the finite value of quark mass. The equation of state of QGP are almost consistent with earlier work, although there is small decrement. Above all, it is verified that the effective quark mass fits nicely in the calculation to produce better results of equation of state of QGP. 


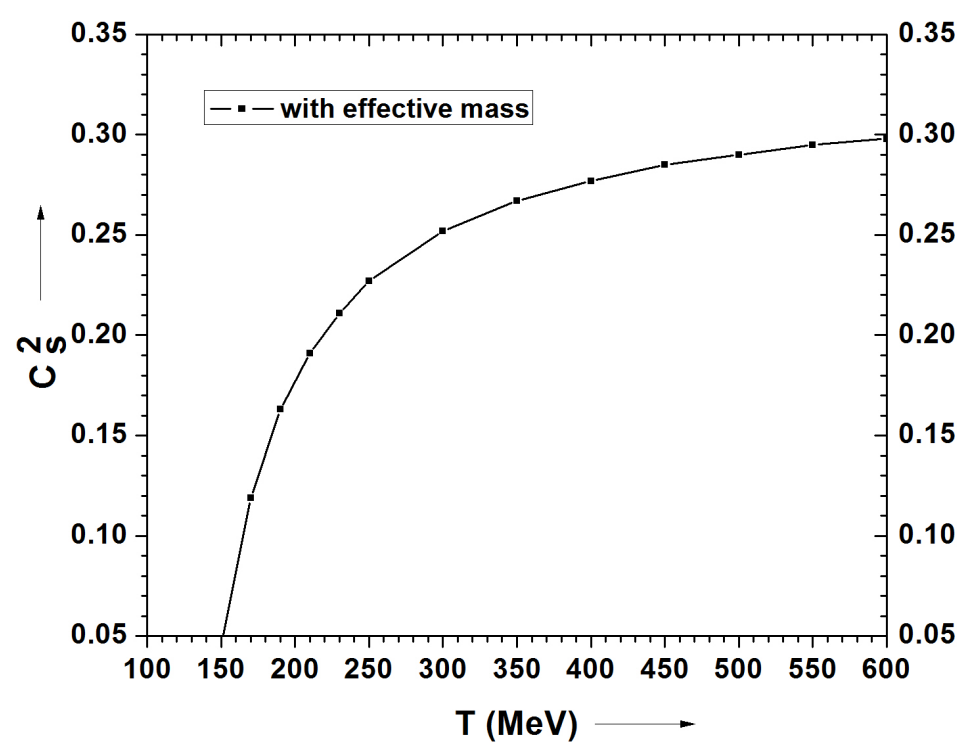

Figure 4. The speed of sound $\left(C_{s}^{2}\right)$ with temperature $(T)$ is plotted

Therefore our results are nearly similar as Ref. [25] using effective quark mass. Finally, this simple phenomenological model does not alter our previous results [25] and provide the same EoS of QGP. The impact of this effective quark mass is very less and hence produce almost same output. The prediction of EoS of QGP by using other models are also in confirmity with recent Lattice QCD results $[4,19,20,22]$.

\section{Conclusion}

Finally we conclude that our model results shows almost similar output with the help of effective quark mass. This model result is successfully applied to the description of the properties of QGP created in the collisions of two massive nuclei at RHIC and LHC. Thus the effective quark mass fitted well with our previous results. The results are also important to produce EoS of QGP and significant in heavy-ion nuclear collisions.

\section{References}

[1] H. Stocker and W. Greiner, Phys. Rept. 137, 277 (1986).

[2] P. Danielewicz, R. Lacey, and W. G. Lynch, Science 298, 1592 (2002).

[3] J. W. Harris and B. M. Müller, Ann. Rev. Nucl. and Par. Sc. 46, 71 (1996).

[4] F. Brau and F. Buisseret, Phys. Rev. D 79, 114007 (2009).

[5] F. Giacosa, Phys. Rev. D 83, 114002 (2011).

[6] S. Borsanyi, et al., JHEP 11, 077 (2010). 
[7] F. Karsch, Nucl. Phys. A 698, 199c-208c (2002).

[8] E. Shuryak, Prog. Part. Nucl. Phys. 62, 48 (2009).

[9] H. Satz, Int. J. Mod. Phys A 21, 672 (2006)

[10] E. V. Shuryak, Phys. Rep. 61, 71 (1980).

[11] J. Cleymans, R. V. Gavai, and E. Suhonen, ibid. 130, 217 (1986).

[12] P. Levai, U. Heinz, Phys. Rev. C 57, 1879 (1998).

[13] R. A. Schneider, W. Weise, Phys. Rev. C 64, 055201 (2001).

[14] A. Peshier, B. Kampfer, O. P. Pavlenko, G. Soff, Phys. Lett. B 337, 235 (1994).

[15] A. Peshier, B. Kampfer, O. P. Pavlenko, G. Soff, Phys. Rev. D 54, 2399 (1996).

[16] Y. Kumar and S. S. Singh, ISRN High E. Phys. 2013, 156747 (2013).

[17] S. S. Singh et al., Pram. J. Phys. 74, 27 (2010).

[18] Y. Kumar, JoP: Conf. Ser. 668, 012110 (2016).

[19] P. K. Srivastava, S. K. Tiwari, C. P. Singh, Phys. Rev. D 82, 014023 (2010).

[20] V. M. Bannur, Eur. Phys. J. C 50, 629 (2007).

[21] M. I. Gorenstein and S. N. Yang, Phys. Rev. D 52, 5206 (1995).

[22] V. M. Bannur, Phys. Lett. B 647, 271 (2007); J. Phys. G: Nucl. Part. Phys. 32, 993 (2006).

[23] V. Goloviznin and H. Satz, Z. Phys. C 57, 671 (1994).

[24] P. Jain, Y. Kumar and D. Kumar, J. Mod. Phys. 5, 792-799 (2014).

[25] Y. Kumar and S. S. Singh, EPJ Web of Conferences 137, 13008 (2017). 